\title{
Formation of Reversible Clusters with Controlled
}

\section{Degree of Aggregation}

\author{
Saba Lotfizadeh, ${ }^{*}$ Hassan Aljama, Dan Reilly, and Themis Matsoukas* \\ Department of Chemical Engineering, The Pennsylvania State University, University Park \\ E-mail: saba.lotfizadeh@gmail.com; matsoukas@engr.psu.edu
}

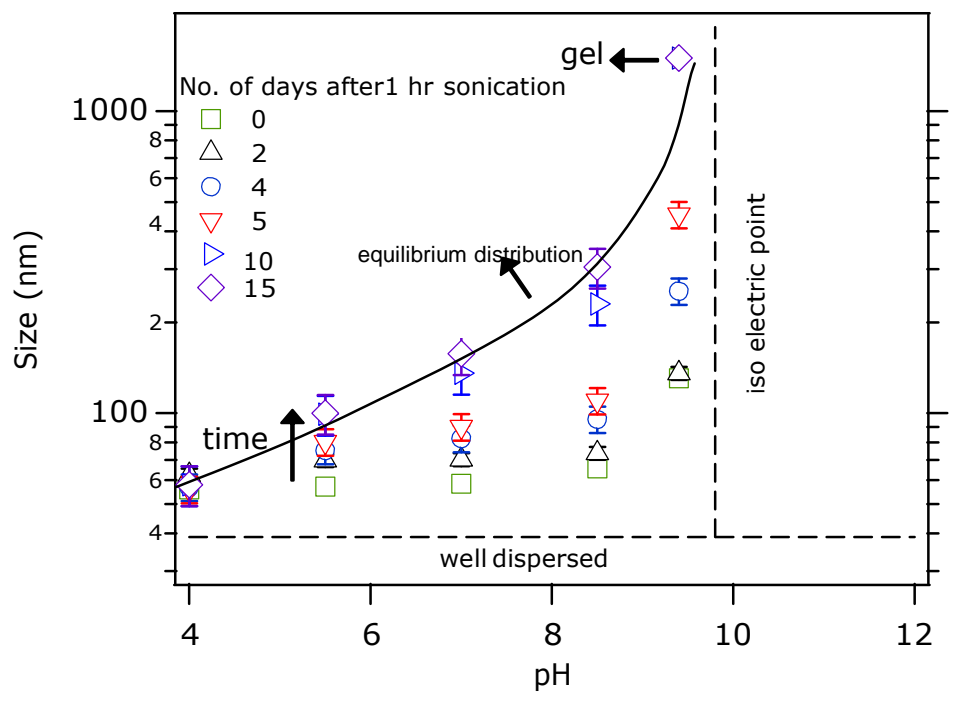

Figure S1: Reversible aggregation responsive to sonication at different $\mathrm{pH}$ values. Samples at each $\mathrm{pH}$ with volume fraction of $22 \%$ were sonicated for 1 hour and size measurements were done afterward daily using DLS measurements. Horizontal dashed lines represent the well dispersed suspension of particles and the gelled suspension, and the red dashed line represents the equilibrium distribution obtained in Figure 4. Particles were sonicated to form smaller aggregates however, as the sonication stops, they grow gradually until the reach their equilibrium distribution and remained at their final size.

${ }^{*}$ To whom correspondence should be addressed 


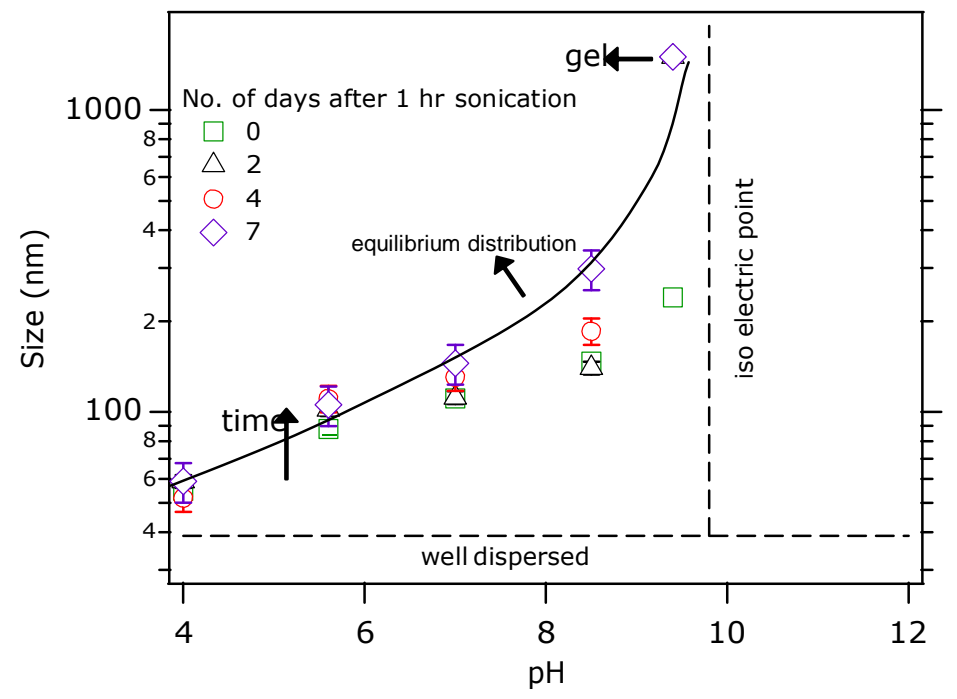

Figure S2: Reversible aggregation responsive to sonication at different $\mathrm{pH}$ values. Samples at each $\mathrm{pH}$ with volume fraction of $6 \%$ were sonicated for 1 hour and size measurements were done afterward daily using DLS measurements. Horizontal dashed lines represent the well dispersed suspension of particles and the gelled suspension, and the red dashed line represents the equilibrium distribution obtained in Figure 4. Particles were sonicated to form smaller aggregates however, as the sonication stops, they grow gradually until the reach their equilibrium distribution and remained at their final size. 